\title{
Implementation of Area optimized Low power Multiplication and Accumulation
}

\author{
S.China Venkateswarlu, N.Uday Kumar, N.Sandeep Kumar, Aannam Karthik, V.Vijay
}

\begin{abstract}
There is number of computations involved at every stage in Digital Signal Processing (DSP). At every stage of computation we have addition and multiplication of the terms derived from previous and presents stages. The general computation incorporates the use of normal multiplication and addition, but the circuitry of normal multiplication and addition is lethargic i.e., it consumes more space on chip, consumes more power and the speed of computation is also low.These drawbacks can be avoided by switching to proposed method called Multiplication and Accumulation (MAC). Aim of this project is to develop an Area optimized Low power digital circuit for MAC (Multiply and Accumulate) operation. We develop the Verilog Hardware Description Language code for the various implementations of the MAC (Multiply and Accumulate) that is we try to avoid using multipliers and prefer to use the combinational circuits like multiplexers. These Verilog HDL codes will be simulated to check the functionality. Once we get the expected results we go for the implementation of the digital circuits. We analyze all the MAC digital circuits to find out the best digital circuit which consumes minimum area and power. The importance of MAC in FPGA designs is explained by some filter designs. We also give some suggestions on the system level solutions based on the MAC.
\end{abstract}

Keywords: Multiplier Accumulator Unit, Digital Signal Processing, Embedded Systems Algorithmic Noise Tolerant, Replica Redundancy Block

\section{INTRODUCTION}

The multiplier and multiplier-accumulator are the essential elements of the digital signal processing for example separating, convolution, and inner products. This unit can compute running total of products, which is at the core of algorithms, for example, the FIR and FFT. The capacity to register with a quick MAC unit is fundamental to accomplish elite in many DSP algorithms, and is the reason there is in any event one MAC unit in all of contemporary profitable DSP processors. Most digital signal processing strategies utilize nonlinear functions for example discrete cosine transform or discrete wavelet transform. In most of digital signal processing applications the basic activities are the multiplication and accumulation. Multiplier-

Revised Manuscript Received on August 05, 2019. Engineering, Affiliated to JNTUH, Hyderabad., undigal, Hyderabad, Telangana,India. (Email: prof.cvsonagiri@gmail.)

Dr.N. Uday Kumar, Professor-ECE, Marri Laxman Institute of Technology, Affiliated to JNTUH, Hyderabad, Dundigal, Hyderabad, Telangana,India. (Email: joyudaya@gmail.com)

N.Sandeep Kumar, Assistant Professor-ECE,Institute of Aeronautical Engineering, Affiliated to JNTUH, Hyderabad, Dundigal, Hyderabad, Telangana,India. (Email: prof.cvsonagiri@gmail.com)

Aannam Karthik, Assistant Professor-ECE,Institute of Aeronautical Engineering, Affiliated to JNTUH, Hyderabad, Dundigal, Hyderabad, Telangana,India. (Email: karthik011190@gmail.com)

Dr.V.Vijay, Professor-ECE,Institute of Aeronautical Engineering, Affiliated to JNTUH, Hyderabad, Dundigal, Hyderabad, Telangana,India (Email: V.vijay@iare.ac.in)
Dr.S.China Venkateswarlu, Professor-ECE,Institute of Aeronautical

Accumulator (MAC) unit consumes low power and is dependably a key to accomplish an elite digital signal processing system. Finite impulse response filters are generally utilized in different DSP applications.

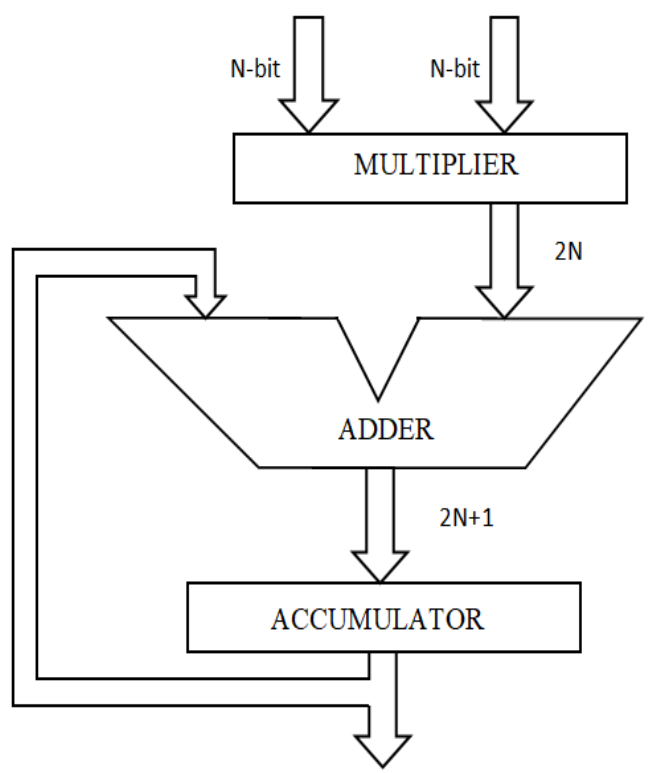

Figure 1.1: Basic Structure of Multiply Accumulate Unit

Another structure has been embraced to expand the throughput rate and special pipeline structures were utilized in the accumulator to decrease the complete latency. Used in the design and implementation of Finite Impulse Response filter utilizing a low power MAC unit with clock gating and pipelining methods to save power. In most of digital signal processing applications the basic activities are the multiplication and accumulation. Multiply-Accumulate unit that devours low power is dependably a key to accomplish an elite in digital signal processing framework. Finite impulse response filters are generally utilized in different DSP applications.

We first design a 1-bit or 2-bit MAC unit, with proper geometries that gives improved power, part and postponement. The postponement in the pipeline organizes in the MAC unit is evaluated dependent on which a control unit is intended to control the information stream between the MAC obstructs for low power. Correspondingly, the $\mathrm{N}$ bit MAC unit is planned and controlled for low power 
utilizing a control rationale that enables the pipelined stages at appropriate time. The viper cell planned has bit of leeway of high operational speed, little transistor tallies and low power. Ripple Carry Adder is for the most part utilized as an accumulator in the plan. This exploration work also likewise examines on different models of multipliers and adders which are appropriate for execution of high throughput signal processing and in the meantime to accomplish low power utilization. The proficiency as far as area and speed of proposed MAC unit architecture is seen through diminished area, low basic postponement and low hardware intricacy. The proposed MAC unit diminishes the area by decreasing the quantity of multiplication and expansion in the multiplier unit. The proposed MAC unit can be executed on a field programmable gate array device. The presentation development results as far as speed and device usage are contrasted with before MAC architecture. Despite the fact that the utilization of Vedic mathematics strategies for multiplication is described in literature, it has been seen that the proposed technique of MAC unit execution is utilizing multiplication unit and shows upgrades in the delay and area. The general development of the MAC task can be displayed by this equation

$$
Z=A \times B+Z
$$

\section{A. Literature Review}

a) MarojuSaiKumar\& D. Ashok Kumar allowed "Design \& Performance Analysis of Multiply-Accumulate (MAC) Unit" grants MAC unit model which is planned by consolidating different multipliers for example, Array multiplier, Ripple carry multiplier through row bypassing method, Wallace multiplier \& DADDA multiplier. Execution of MAC unit stands examined in standings of Area, delay and power. Proposed MAC unit model (DM+CSA+Acc) accomplishes improved execution as far as area and delay contrasted with current technique. In any case, it has small growth in power. The presentation investigation of MAC unit models is finished by planning models in Verilog HDL. At that time MAC unit models remain replicated \& produced in Xilinx ISE 13.2 fir Virtex6 family $40 \mathrm{~nm}$ technology.

b) Thirumala Rao V., Girish Gandhi S. \&Leela Mohan C. permitted "Performance Evaluation of Parallel Multipliers for High Speed MAC Design" that presented execution of high speed Signed \& Unsigned fast Multipliers \& their relative investigation. Planned manner for broadly utilized parallel multipliers for example, Booth multiplier, Wallace multiplier and DADDA Tree multiplier all together get the plan qualities like Speed, area. The MAC executed utilizing Wallace Multiplier had slightest interruption when contrasted with others. The developed design constraints of multipliers were examined to structure optimum speed multiply and Accumulate (MAC) unit for multimedia application like Filters, Synthesizers,

\section{B. Problem Identification}

Though there are many ways by means of which MAC unit has been implemented, out of which some are by using specific multipliers and adders. We have many good multipliers such as Wallace Tree Multiplier, Booth Multiplier, etc. Also we have many adders like Ripple carry
Adder, Carry Look Ahead Adder, etc., but inspite of that we observed little flaws in their operations. There is specifically no issue with the Adders, but the issue lies with the Multipliers. The multiplication operation in those cases are carried out by using asterisk “*”, which is nothing but called a star operator used for multiplication.

Hence it is required to counter this big flaw. Hence we came up with an idea of making the Multiplier Less Algorithm for carrying out the multiplication operation. As we know that any program will work extremely fast if it is incorporated with the processor level commands. We made the use of this fact and developed a unit MAC, which is actually multiplier less unit.

\section{METHODOLOGY}

A key part of study or hypothesis is the methodology. This isn't exactly equivalent to "methods". The methodology portrays the wide philosophical supporting to your picked research methods, including whether you are utilizing qualitative or quantitative methods, or a blend of both, and why.

\section{A. METHODOLOGY OF EXISTING METHODS}

In 2 x 2-bit multiplier, the multiplicand has two bits each and result of multiplication is of four bits. Input ranges from " 00 " to " $11 "$ and the output lies in the set of " 0000 " to " $1111 "$ " "00" to " 11 " and the output lies in the set of " $0000 "$ to "1111". Figure 1.1 shows the stepwise multiplication of two binary numbers using Vedic mathematics technique.

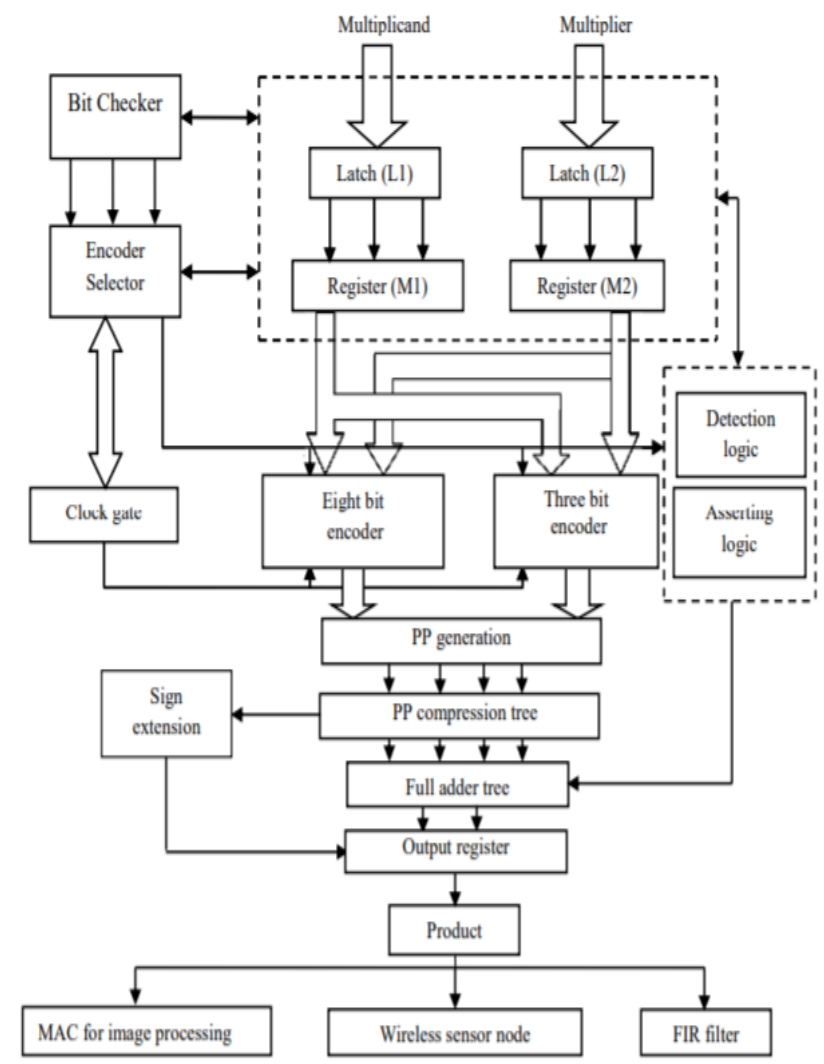

Figure 2.1: Block Diagram of Developed Multiplier

Blue Eyes Intelligence Engineering

\& Sciences Publication 


\section{METHODOLOGY OF PROPOSED METHOD}

The basic operation in most of the Digital Signal processing applications is MAC. The proposed method is on design and development of an area optimized MAC (Multiply and Accumulate Block).Proposed method involves: a) Developing the traditional multiplier-based MAC, synthesis and area calculation. b) Developing a windows-based application to generate the multiplier less MAC equations. c) Developing the various multipliers less MAC blocks. d) Synthesis of all the multiplier less MAC blocks. e) Summarizing the area utilization of all the multiplier less MAC blocks and comparing them with the traditional multiplier-based MAC. f) Explain the importance of the proposed method in any of the real time applications like Video processing systems.

\section{IMPLEMENTATION}

In this section different techniques and methodologies have been discussed by means of which we have implemented our Research work. Multiplier Based Traditional Method,

Multiplier Less Mac Method 1. In Multiplier less method I, we have implemented MAC using Multiplier less algorithm. To make it multiplier less we have used shifting operations “《”, “〉》" instead of using asterisk “*”. Since the shifting commands get executed at the processor levels, they are very much fast as compared to the asterisk operator and also they consume few processor resources which had been proved in the later section. We have designed a 4-bit coefficient and 8-bit variable MAC. Initially we designed a $\mathrm{C}$-program by which we generated the MAC equations, i.e., we wrote the $\mathrm{C}$-code that generates the MAC equations when given the length of coefficients.

The addition program using "+" operator has been written using Verilog language on Mentor Graphics ModelSimTool, and later is synthesized using Synopsys Synplify Pro Synthesis Tool in order to generate the resource consumption report. Below is the resource utilization report generated by using Synopsys Synplify Pro Synthesis Tool.

\section{SIMULATION RESULTS}

In this research work simulation waveforms of proposed method (or) ideology has been included. Apart from that an explanation related to the functionality as per the waveforms has also been provided.INPUTS:4-bit coeff, 8-bit var, clk, rst,

OUTPUTS: macout Multiplication Algorithm Multiplier Less Algorithm-I Here initially we have considered Multiplier Less Algorithm-I (MLA-I) from the implementation section. MLA-I has been simulated and its snippet has been included. The waveforms have also been explained briefly.Apart from its simulation, synthesizing has also been done by using Synopsys Synplify Pro Synthesis tool and the snippet has been attached below.CASE-I: When rst $=-1$. The input provided is 4-bit coeff as: "1110" whose value in decimal is "14" and 8-bit var as: "0101011110" whose value in decimal is " 86 ". Here it is observed clearly that when $r s t=1$, then irrespective of any inputs the output is zero "000000000000" (12'b0).In short the system is reset. Now let us see when $r s t=0$. Observed Simulation waveform of MLA-I when rst $=0$ Here we have made $r s t=$ 0 , so basically now the system is out of reset state, hence the system will function as it was designed to function. Now it will produce the output which will be the multiplication of both the inputs.Clearly the output obtained is macout= "010010110100" whose decimal value is " 1204 ".By this the functionality of our proposed idea is verified. Below shown is the snippet which is the synthesis outcome of this particular algorithm MLA-I using Synopsys Synlipy Pro Synthesis Tool.

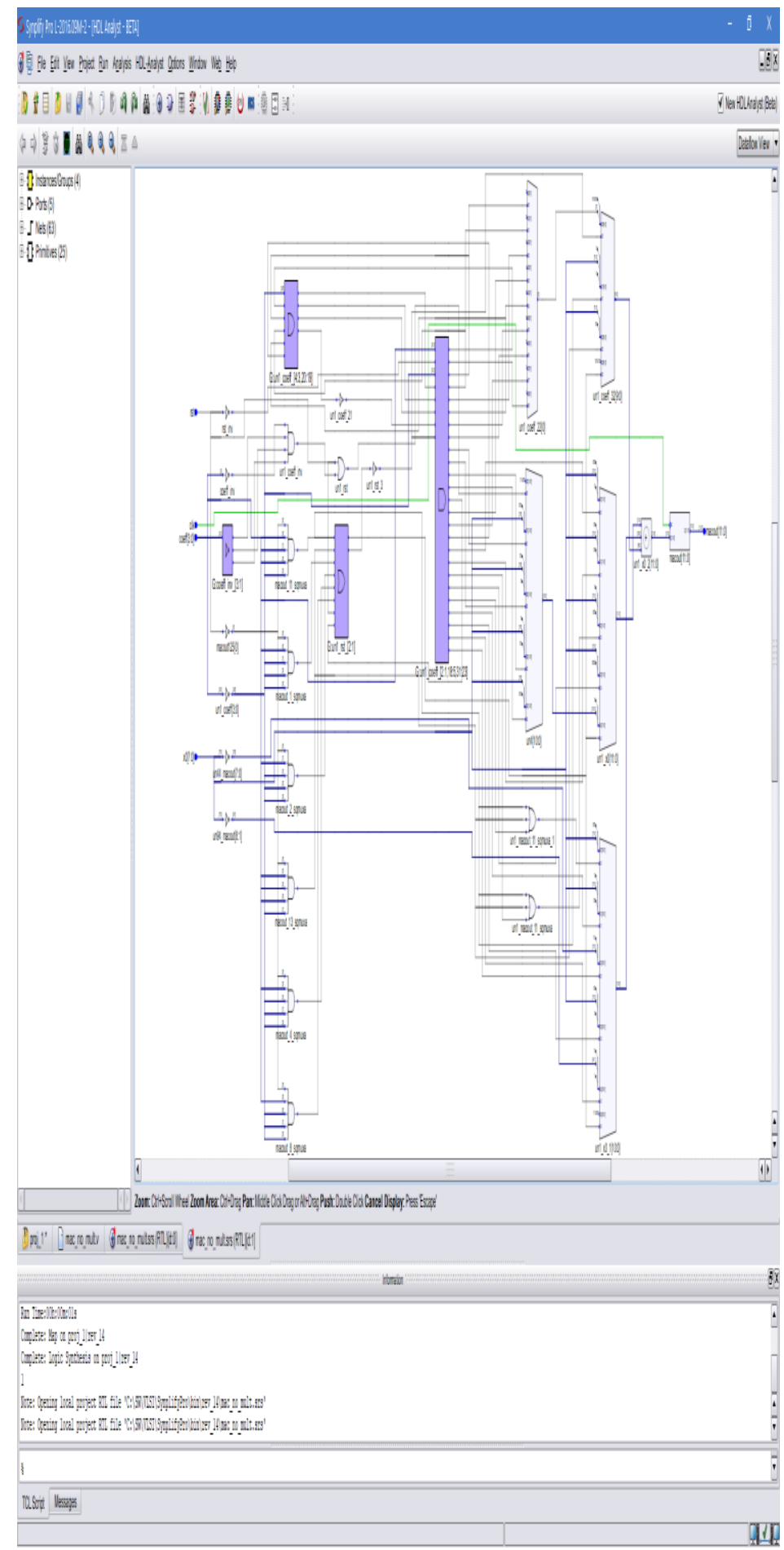

Figure 5.1: Synthesis Outcome of MLA-I

Published By:

Blue Eyes Intelligence Engineering

\& Sciences Publication 


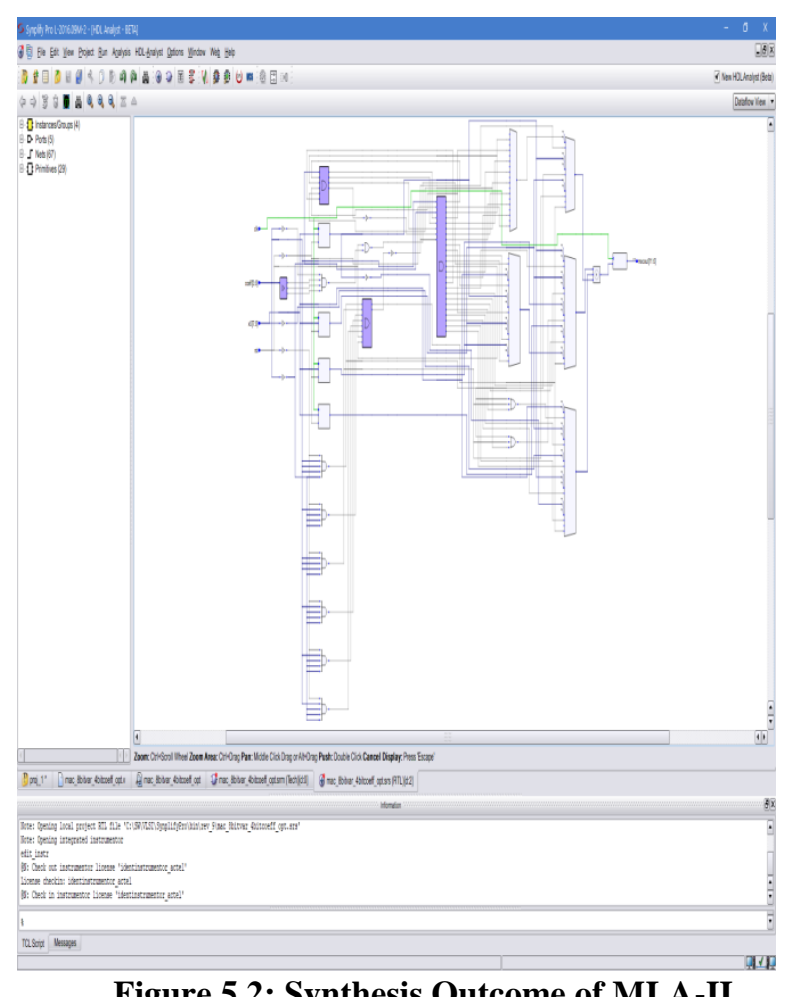

Figure 5.2: Synthesis Outcome of MLA-II

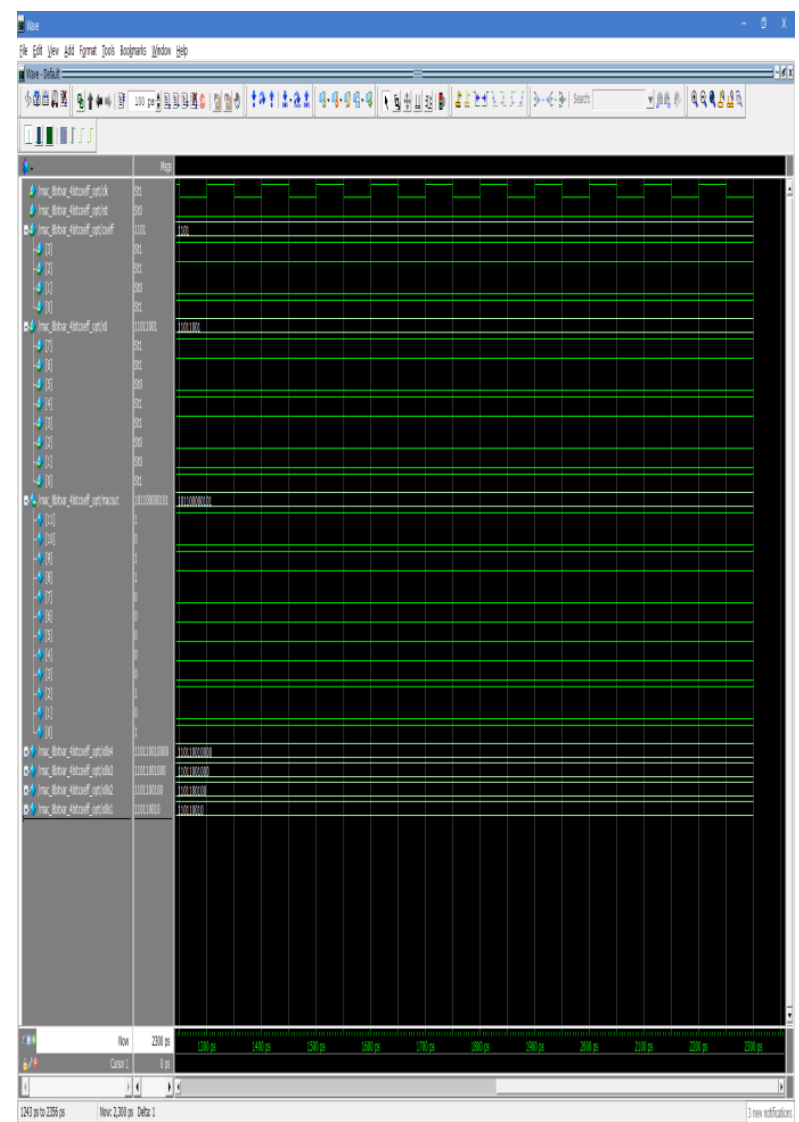

Figure 5.3: Simulation waveform of MLA-II when $\mathbf{r s t}=\mathbf{0}$

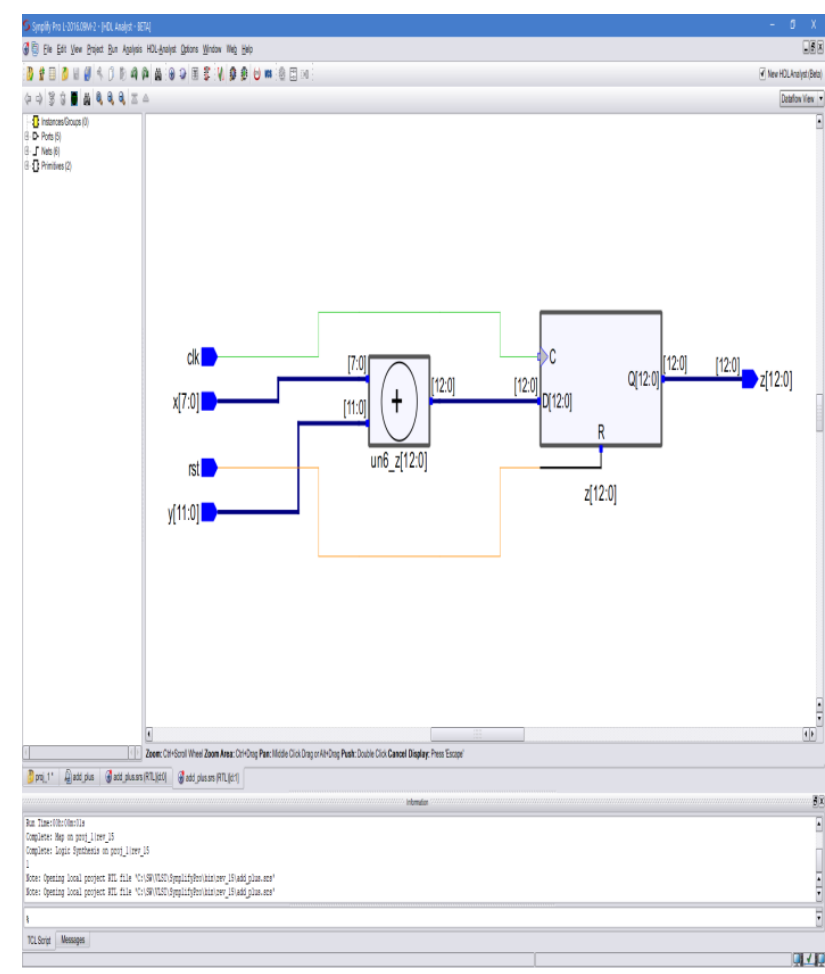

Figure 5.4: Synthesis Outcome of Adder implemented using “+” operatoron Synopsys Synplify Pro Synthesis Tool

\section{CONCLUSION AND FUTURE SCOPE}

The electronics and semiconductor industry involve a critical spot in modern culture by helping human desires and sub-serving human happiness. Therefore, with the progression of time and evolution in demand for electronic gadgets, the industry has seen captivating development. The advancement has been increasingly articulated in the field of convenient specialized gadgets like Mobile phones, IPADS and notepads. The current elite frameworks utilized in the correspondence framework uses Multiply Accumulate block which expends enormous area and power and are described by higher information throughput rate. However, in the power-starved society, there is a basic need to find frameworks that can reduce power and rise the speed of MAC system. In this specific circumstance, a few MAC with its area and power decrease procedures have been engaged in this project effort. In addition, this investigation would assist the future specialists with exploring more in the field of low power, area and high speed multiply-accumulate unit.

The proposed methodology is designed keeping computational operations performed in Digital Signal Processing applications in mind, but this can be further increased to other domains like Video Processing, and interestingly in Image Processing. The main aim of MAC unit is actually the pace at which an operation is performed i.e., speed of operation. With further enhancements in the proposed methodology we can make MAC unit as more area efficient, faster, and minimize the power consumption even more. They can be used in the implementation of finite

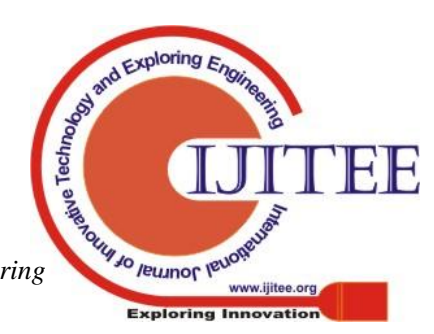


impulse response filter design for DSP which are advantageous for low power applications. ASIC design for low power digital filter with low latency and power gating can be carried out. These arithmetic sub systems can be used in the implementation of arithmetic and logic units and multiply and accumulate units for DSP processor which improves performance of the system in different level of abstraction. The GDI based counter designs can be improved by using clock swing techniques in flip flops.

\section{APPENDIX}

Appendix A: Structure of Multiply Accumulate Unit , Developed Multiplier, Synthesis Outcome of MLA-I, Synthesis Outcome of MLA-II, Simulation waveform of MLA-II when rs values varies from 0 to and so on 0 , Synthesis Outcome of Adder implemented using using "+" operation Synopsys Simplify Pro Synthesis Tool.

\section{ACKNOWLEDGMENT}

The work is carried out through the research facility at the Department of Electronics \& Communication Engineering, Institute of Aeronautical Engineering, Dundigal, Hyderabad, Telangana, INDIA. Department of Electronics \& Communication Engineering, MLRITM, Hyderabad. The Authors also would like to thank the authorities of JNTUH, for encouraging this research work.

\section{REFERENCES}

1 Pratap Kumar Dakua, Anamika Sinha, Shivdhari\&Gourab,"Hardware Implementation of MAC unit," International Journal of Electronics Communication and Computer Engineer ing, vol. 3, 2012.

2 M.Jeevitha, R.Muthaiah, P.Swaminathan, "Review Article: Efficient Multiplier Architecure in VLSI Design," Journal of Theoretical and Applied Information Technology, vol. 38, no. 2, April 2012.

3 Ravi Shankar Mishra, PuranGour, Braj Bihari Soni, "Design and Implements of Booth and Robertson's multipliers algorithm on FPGA," International Journal of EngineeringResearch and Applications, Vol. 1, Issue 3, pp. 905-910, 2011.

4 Tung Thanh Hoang, Magnus Själander, Per LarssonEdefors, "A High-Speed, Energy-Efficient TwoCycle Multiply-Accumulate (MAC) Architecture and Its Application to aDouble-Throughput MAC Unit," IEEE transactions on Circuits \& Systems, vol. 57, no. 12, pp. 3073-3081, Dec. 2010.

5 A. Abdelgawad, MagdyBayoumi, "High Speed andAreaEfficient Multiply Ac cumulate (MAC) Unit for DigitalSignal Prossing Applications,” IEEE International Symposium on Circuits \&Systems , pp. 3199 - 3202, 2007.

6 Berkeley Design Technology, Inc., "Choosing a DSP Processor," World Wide Web, http://www.bdti.com/articles/choose_2000.pdf, 2000.

7 Jennifer Eyre and Jeff Bier, "The Evolution of DSP Processors", Berkeley Design Technol ogy,Inc.,http://www.bdti.com/articles/evolution.pdf, 2000.

\section{AUTHORS PROFILE}

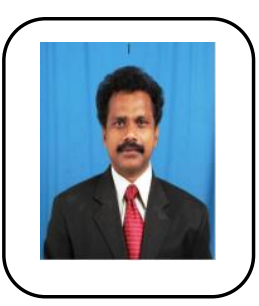

Dr.S.China Venkateswarlu, He obtained his B.Tech (ECE), M.Tech (DSCE), $\mathrm{Ph} . \mathrm{D}(\mathrm{DSP})$, working as a Professor in Dept.of ECE at Institute of Aeronautical Engineering, Hyderabad.TG, INDIA. He is having more than 22 years of teaching experience and published more than 25 papers in referred National and International Journals. He has presented more than 15 papers in National and International Journals. He has reviewed one Book on Digital Signal Processing from M/S Persons Educations. He is a IEEE Member, Life member of ISTE, CSI, IAENG, SDIWC, ISRD, IFERP. He is working as a Reviewer in Different International Journals Elsevier-Signal Processing. Edas. His areas of interest Digital Signal Processing, Speech Processing, ,Hearing Aid, Embedded Systems, Digital Image Processing.

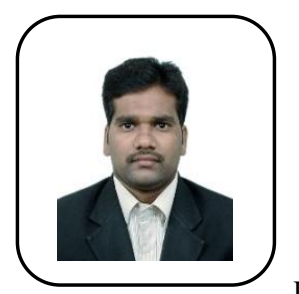

Dr.N.Uday Kumar, He obtained his B.Tech,M.Tech,Ph.D., working as a Professor in Dept.of ECE at Marri Laxman Reddy Institute of Technology \& Management,a, Hyderabad.TG, INDIA. He is having more than 10 years of teaching experience and published more than 6 papers in referred National and International Journals. He has presented more than 9 apers in National and International Journals. He is a Life member of MIE and IAENG. His areas of interest Embedded Systems, Digital Image Processing.

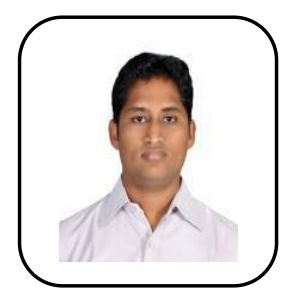

N.Sandeep Kumar, He obtained his B.Tech M.Tech, working as a Assistant Professor in Dept.of ECE at Institute of Aeronautical Engineering, Hyderabad.TG, INDIA. He is having more than 7 years of teaching experience and published more than 6 papers in referred National and International Journals. He has presented more than 5 papers in National and International Journals.. His areas of interest Digital Signal Processing, Speech Processing, ,Hearing Aid, Embedded Systems, Digital Image Processing.

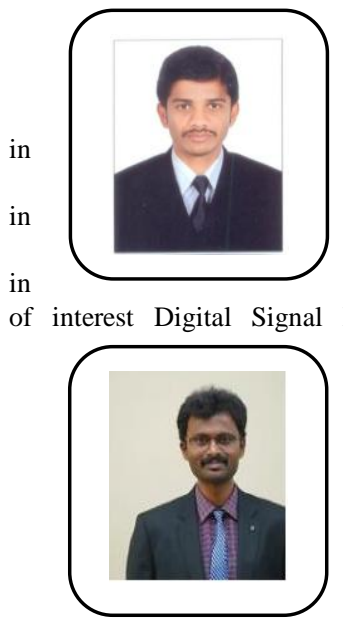

A.KARTHIK Research scholar in department of ECE-Veltech Dr.RR \& Dr.Sr University,Chennai. M.Tech (ECE) Mallareddy institute of technology \& science (JNTUH), Obtained B.Tech (ECE) Vaageswari College of engineering (JNTUH) .Worked as Assistant professor Narsimha reddy engineering college. Areas Processing, Speech processing, Embedded Systems,karthik011190@gmail.com.

Dr.V.Vijay, He obtained his B.Tech, M.Tech , Ph.D, working as a Professor in Dept.of ECE at Institute of Aeronautica Engineering, Hyderabad.TG, INDIA. He is having more than 15 years of teaching experience and published more than 20 papers in referred National and International Journals. He has presented more than 10 papers in National and International Journals. He is a IEEE Member, ISTE, IAENG, His areas of interest VLSI, Signal Processing, ,Hearing Aid. 